\title{
ON SOME PARTITIONS OF A FLAG MANIFOLD*
}

\author{
GEORGE LUSZTIG ${ }^{\dagger}$ \\ Dedicated to Professor Dan Papuc on his 80th birthday
}

Key words. Reductive group, Weyl group, flag manifold, unipotent class.

AMS subject classifications. 20 G99.

Introduction. Let $G$ be a connected reductive group over an algebraically closed field $\mathbf{k}$ of characteristic $p \geq 0$. Let $\mathbf{W}$ be the Weyl group of $G$. Let $\underline{\mathbf{W}}$ be the set of conjugacy classes in $\mathbf{W}$. The main purpose of this paper is to give a (partly conjectural) definition of a surjective map from $\mathbf{W}$ to the set of unipotent classes in $G$ (see 1.2(b)). When $p=0$, a map in the opposite direction was defined in [KL, 9.1] and we expect that it is a one sided inverse of the map in the present paper. The (conjectural) definition of our map is based on the study of certain subvarieties $\mathcal{B}_{g}^{w}$ (see below) of the flag manifold $\mathcal{B}$ of $G$ indexed by a unipotent element $g \in G$ and an element $w \in \mathbf{W}$.

Note that $\mathbf{W}$ naturally indexes $\left(w \mapsto \mathcal{O}_{w}\right)$ the orbits of $G$ acting on $\mathcal{B} \times \mathcal{B}$ by simultaneous conjugation on the two factors. For $g \in G$ we set $\mathcal{B}_{g}=\{B \in \mathcal{B} ; g \in B\}$. The varieties $\mathcal{B}_{g}$ play an important role in representation theory and their geometry has been studied extensively. More generally for $g \in G$ and $w \in \mathbf{W}$ we set

$$
\mathcal{B}_{g}^{w}=\left\{B \in \mathcal{B} ;\left(B, g B g^{-1}\right) \in \mathcal{O}_{w}\right\} .
$$

Note that $\mathcal{B}_{g}^{1}=\mathcal{B}_{g}$ and that for fixed $g,\left(\mathcal{B}_{g}^{w}\right)_{w \in \mathbf{W}}$ form a partition of the flag manifold $\mathcal{B}$.

For fixed $w$, the varieties $\mathcal{B}_{g}^{w}(g \in G)$ appear as fibres of a map to $G$ which was introduced in [L3] as part of the definition of character sheaves. Earlier, the varieties $\mathcal{B}_{g}^{w}$ for $g$ regular semisimple appeared in [L1] (a precursor of [L3]) where it was shown that from their topology (for $\mathbf{k}=\mathbf{C}$ ) one can extract nontrivial information about the character table of the corresponding group over a finite field.

I thank David Vogan for some useful discussions.

\section{The sets $\mathbf{S}_{g}$.}

1.1. We fix a prime number $l$ invertible in $\mathbf{k}$. Let $g \in G$ and $w \in \mathbf{W}$. For $i, j \in \mathbf{Z}$ let $H_{c}^{i}\left(\mathcal{B}_{g}^{w}, \overline{\mathbf{Q}}_{l}\right)_{j}$ be the subquotient of pure weight $j$ of the $l$-adic cohomology space $H_{c}^{i}\left(\mathcal{B}_{g}^{w}, \overline{\mathbf{Q}}_{l}\right)$. The centralizer $Z(g)$ of $g$ in $G$ acts on $\mathcal{B}_{g}^{w}$ by conjugation and this induces an action of the group of components $\bar{Z}(g)$ on $H_{c}^{i}\left(\mathcal{B}_{g}^{w}, \overline{\mathbf{Q}}_{l}\right)$ and on each $H_{c}^{i}\left(\mathcal{B}_{g}^{w}, \overline{\mathbf{Q}}_{l}\right)_{j}$. For $z \in \bar{Z}(g)$ we set

$$
\Xi_{g, z}^{w}=\sum_{i, j \in \mathbf{Z}}(-1)^{i} \operatorname{tr}\left(z, H_{c}^{i}\left(\mathcal{B}_{g}^{w}, \overline{\mathbf{Q}}_{l}\right)_{j}\right) v^{j} \in \mathbf{Z}[v]
$$

where $v$ is an indeterminate; the fact that this belongs to $\mathbf{Z}[v]$ and is independent of the choice of $l$ is proved by an argument similar to that in the proof of [DL, 3.3].

\footnotetext{
* Received January 26, 2010; accepted for publication October 21, 2010.

† Department of Mathematics, M.I.T., Cambridge, MA 02139, USA (gyuri@math.mit.edu). Supported in part by the National Science Foundation.
} 
Let $l: \mathbf{W} \rightarrow \mathbf{N}$ be the standard length function. The simple reflections $s \in \mathbf{W}$ (that is the elements of length 1 of $\mathbf{W}$ ) are numbered as $s_{1}, s_{2}, \ldots$ Let $w_{0}$ be the element of maximal length in $\mathbf{W}$.

Let $\mathcal{H}$ be the Iwahori-Hecke algebra of $\mathbf{W}$ with parameter $v^{2}$ (see [GP, 4.4.1]; in the definition in loc.cit. we take $\left.A=\mathbf{Z}\left[v, v^{-1}\right], a_{s}=v^{2}, b_{s}=v^{2}-1\right)$. Let $\left(T_{w}\right)_{w \in \mathbf{W}}$ be the standard basis of $\mathcal{H}$ (see [GP, 4.4.3, 4.4.6]). For $w \in \mathbf{W}$ let $\hat{T}_{w}=v^{-2 l(w)} T_{w}$. If $s_{i_{1}} s_{i_{2}} \ldots s_{i_{t}}$ is a reduced expression for $w \in \mathbf{W}$ we write also $\hat{T}_{w}=\hat{T}_{i_{1} i_{2} \ldots i_{t}}$.

For any $g \in G, z \in \bar{Z}(g)$ we set

$$
\Pi_{g, z}=\sum_{w \in \mathbf{W}} \Xi_{g, z}^{w} \hat{T}_{w} \in \mathcal{H}
$$

The following result can be proved along the lines of the proof of [DL, Theorem 1.6] (we replace the Frobenius map in that proof by conjugation by $g$ ); alternatively, for $g$ unipotent, we may use 1.5(a).

(a) $\Pi_{g, z}$ belongs to the centre of the algebra $\mathcal{H}$.

According to [GP, 8.2.6, 7.1.7], an element $c=\sum_{w \in \mathbf{W}} c_{w} \hat{T}_{w}\left(c_{w} \in \mathbf{Z}\left[v, v^{-1}\right]\right)$ in the centre of $\mathcal{H}$ is uniquely determined by the coefficients $c_{w}\left(w \in \mathbf{W}_{\text {min }}\right)$ and we have $c_{w}=c_{w^{\prime}}$ if $w, w^{\prime} \in \mathbf{W}_{\text {min }}$ are conjugate in $\mathbf{W}$; here $\mathbf{W}_{\text {min }}$ is the set of elements of $\mathbf{W}$ which have minimal length in their conjugacy class. This applies in particular to $c=\Pi_{g, z}$, see (a). For any $C \in \underline{\mathbf{W}}$ we set $\Xi_{g, z}^{C}=\Xi_{g, z}^{w}$ where $w$ is any element of $C \cap \mathbf{W}_{\min }$.

Note that if $g=1$ then $\Pi_{g, 1}=\left(\sum_{w} v^{2 l(w)}\right) 1$. If $g$ is regular unipotent then $\Pi_{g, 1}=\sum_{w \in \mathbf{W}} v^{2 l(w)} \hat{T}_{w}$. If $G=P G L_{3}(\mathbf{k})$ and $g \in G$ is regular semisimple then $\Pi_{g, 1}=6+3\left(v^{2}-1\right)\left(\hat{T}_{1}+\hat{T}_{2}\right)+\left(v^{2}-1\right)^{2}\left(\hat{T}_{12}+\hat{T}_{21}\right)+\left(v^{6}-1\right) \hat{T}_{121}$; if $g \in G$ is a transvection then $\Pi_{g, 1}=\left(2 v^{2}+1\right)+v^{4}\left(\hat{T}_{1}+\hat{T}_{2}\right)+v^{6} \hat{T}_{121}$.

For $g \in G$ let $\operatorname{cl}(g)$ be the $G$-conjugacy class of $g$; let $\overline{c l(g)}$ be the closure of $c l(g)$. Let $\mathbf{S}_{g}$ be the set of all $C \in \underline{\mathbf{W}}$ such that $\Xi_{g, 1}^{C} \neq 0$ and $\Xi_{g^{\prime}, 1}^{C}=0$ for any $g^{\prime} \in \overline{c l(g)}-\operatorname{cl}(g)$. If $\mathcal{C}$ is a conjugacy class in $G$ we shall also write $\mathbf{S}_{\mathcal{C}}$ instead of $\mathbf{S}_{g}$ where $g \in \mathcal{C}$.

We describe the set $\mathbf{S}_{g}$ and the values $\Xi_{g, 1}^{C}$ for $C \in \mathbf{S}_{g}$ for various $G$ of low rank and various unipotent elements $g$ in $G$. We denote by $u_{n}$ a unipotent element of $G$ such that $\operatorname{dim} \mathcal{B}_{u_{n}}=n$. The conjugacy class of $w \in \mathbf{W}$ is denoted by $(w)$.

$G$ of type $A_{1}$.

$$
\mathbf{S}_{u_{1}}=(1), \mathbf{S}_{u_{0}}=\left(s_{1}\right) ; \Xi_{u_{1}, 1}^{1}=1+v^{2}, \Xi_{u_{0}, 1}^{s_{1}}=v^{2} .
$$

$G$ of type $A_{2}$.

$$
\begin{gathered}
\mathbf{S}_{u_{3}}=(1), \mathbf{S}_{u_{1}}=\left(s_{1}\right), \mathbf{S}_{u_{0}}=\left(s_{1} s_{2}\right) . \\
\Xi_{u_{3}, 1}^{1}=1+2 v^{2}+2 v^{4}+v^{6}, \Xi_{u_{1}, 1}^{\left(s_{1}\right)}=v^{4}, \Xi_{u_{0}, 1}^{\left(s_{1} s_{2}\right)}=v^{4} .
\end{gathered}
$$

$G$ of type $B_{2}, p \neq 2$. (The simple reflection corresponding to the long root is denoted by $s_{1}$.)

$$
\begin{aligned}
& \mathbf{S}_{u_{4}}=(1), \mathbf{S}_{u_{2}}=\left(s_{1}\right), \mathbf{S}_{u_{1}}=\left\{\left(s_{2}\right),\left(s_{1} s_{2} s_{1} s_{2}\right)\right\}, \mathbf{S}_{u_{0}}=\left(s_{1} s_{2}\right) . \\
& \quad \Xi_{u_{4}, 1}^{1}=\left(1+v^{2}\right)^{2}\left(1+v^{4}\right), \Xi_{u_{2}, 1}^{\left(s_{1}\right)}=v^{4}\left(1+v^{2}\right), \Xi_{u_{1}, 1}^{\left(s_{2}\right)}=2 v^{4} \\
& \Xi_{u_{1}, 1}^{\left(s_{1} s_{2} s_{1} s_{2}\right)}=v^{6}\left(v^{2}-1\right), \Xi_{u_{0}, 1}^{\left(s_{1} s_{2}\right)}=v^{4}
\end{aligned}
$$


$G$ of type $B_{2}, p=2$. ( $u_{2}^{\prime}$ denotes a transvection; $u_{2}^{\prime \prime}$ denotes a unipotent element with $\operatorname{dim} \mathcal{B}_{u_{2}^{\prime \prime}}=2$ which is not conjugate to $u_{2}^{\prime}$.)

$$
\begin{aligned}
& \mathbf{S}_{u_{4}}=(1), \mathbf{S}_{u_{2}^{\prime}}=\left(s_{1}\right), \mathbf{S}\left(u_{2}^{\prime \prime}\right)=\left(s_{2}\right), \mathbf{S}_{u_{1}}=\left(s_{1} s_{2} s_{1} s_{2}\right), \mathbf{S}_{u_{0}}=\left(s_{1} s_{2}\right) . \\
& \Xi_{u_{4}, 1}^{1}=\left(1+v^{2}\right)^{2}\left(1+v^{4}\right), \Xi_{u_{2}^{\prime}, 1}^{\left(s_{1}\right)}=v^{4}\left(1+v^{2}\right), \Xi_{u_{2}^{\prime \prime}, 1}^{\left(s_{2}\right)}=v^{4}\left(1+v^{2}\right), \\
& \Xi_{u_{1}, 1}^{\left(s_{1} s_{2} s_{1} s_{2}\right)}=v^{8}, \Xi_{u_{0}, 1}^{\left(s_{1} s_{2}\right)}=v^{4} .
\end{aligned}
$$

$G$ of type $G_{2}, p \neq 2,3$. (The simple reflection corresponding to the long root is denoted by $s_{2} \cdot$ )

$$
\begin{aligned}
& \mathbf{S}_{u_{6}}=(1), \mathbf{S}_{u_{3}}=\left(s_{2}\right), \mathbf{S}_{u_{2}}=\left\{\left(s_{1}\right),\left(s_{1} s_{2} s_{1} s_{2} s_{1} s_{2}\right)\right\}, \mathbf{S}_{u_{1}}=\left(s_{1} s_{2} s_{1} s_{2}\right), \\
& \mathbf{S}_{u_{0}}=\left(s_{1} s_{2}\right) . \\
& \Xi_{u_{6}, 1}^{1}=\left(1+v^{2}\right)^{2}\left(1+v^{4}+v^{8}\right), \Xi_{u_{3}, 1}^{\left(s_{2}\right)}=v^{6}\left(1+v^{2}\right), \Xi_{u_{2}, 1}^{\left(s_{1}\right)}=v^{4}\left(1+v^{2}\right), \\
& \Xi_{u_{2}, 1}^{\left(s_{1} s_{2} s_{1} s_{2} s_{1} s_{2}\right)}=v^{8}\left(v^{4}-1\right), \Xi_{u_{1}, 1}^{\left(s_{1} s_{2} s_{1} s_{2}\right)}=2 v^{8}, \Xi_{u_{0}, 1}^{\left(s_{1} s_{2}\right)}=v^{4} .
\end{aligned}
$$

$G$ is of type $A_{3}$. (The simple reflections are $s_{1}, s_{2}, s_{3}$ with $s_{1} s_{3}=s_{3} s_{1}$ ).

$$
\begin{aligned}
& \mathbf{S}_{u_{6}}=(1), \mathbf{S}_{u_{3}}=\left(s_{1}\right), \mathbf{S}_{u_{2}}=\left(s_{1} s_{3}\right), \mathbf{S}_{u_{1}}=\left(s_{1} s_{2}\right), \mathbf{S}_{u_{0}}=\left(s_{1} s_{2} s_{3}\right) . \\
& \Xi_{u_{6}, 1}^{1}=\left(1+v^{2}\right)\left(1+v^{2}+v^{4}\right)\left(1+v^{2}+v^{4}+v^{6}\right), \Xi_{u_{3}, 1}^{\left(s_{1}\right)}=v^{6}+v^{8}, \\
& \Xi_{u_{2}, 1}^{\left(s_{1} s_{3}\right)}=v^{6}+v^{8}, \Xi_{u_{1}, 1}^{\left(s_{1} s_{2}\right)}=v^{6}, \Xi_{u_{0}, 1}^{\left(s_{1} s_{2} s_{3}\right)}=v^{6} .
\end{aligned}
$$

$G$ of type $B_{3}, p \neq 2$. (The simple reflection corresponding to the short root is denoted by $s_{3}$ and $\left(s_{1} s_{3}\right)^{2}=1$.)

$$
\begin{aligned}
\mathbf{S}_{u_{9}} & =(1), \mathbf{S}_{u_{5}}=\left(s_{1}\right), \mathbf{S}_{u_{4}}=\left\{\left(s_{3}\right),\left(s_{2} s_{3} s_{2} s_{3}\right)\right\}, \mathbf{S}_{u_{3}}=\left\{\left(s_{1} s_{3}\right),\left(w_{0}\right)\right\}, \\
\mathbf{S}_{u_{2}} & =\left(s_{1} s_{2}\right), \mathbf{S}_{u_{1}}=\left\{\left(s_{2} s_{3}\right),\left(s_{2} s_{3} s_{1} s_{2} s_{3}\right)\right\}, \mathbf{S}_{u_{0}}=\left(s_{1} s_{2} s_{3}\right) . \\
& \Xi_{u_{9}, 1}^{1}=\left(1+v^{2}\right)^{3}\left(1+v^{4}\right)\left(1+v^{4}+v^{8}\right), \Xi_{u_{5}, 1}^{\left(s_{1}\right)}=v^{8}\left(1+v^{2}\right)^{2}, \\
& \Xi_{u_{4}, 1}^{\left(s_{2} s_{3} s_{2} s_{3}\right)}=v^{8}\left(1+v^{2}\right)\left(v^{4}-1\right), \Xi_{u_{4}, 1}^{\left(s_{3}\right)}=2 v^{6}\left(1+v^{2}\right)^{2}, \\
& \Xi_{u_{3}, 1}^{\left(s_{1} s_{3}\right)}=v^{8}\left(1+v^{2}\right), \Xi_{u_{3}, 1}^{\left(w_{0}\right)}=v^{14}\left(v^{4}-1\right), \Xi_{u_{2}, 1}^{\left(s_{1} s_{2}\right)}=2 v^{8}, \\
& \Xi_{u_{1}, 1}^{\left(s_{2} s_{3}\right)}=2 v^{6}, \Xi_{u_{1}, 1}^{\left(s_{2} s_{3} s_{1} s_{2} s_{3}\right)}=v^{8}\left(v^{2}-1\right), \Xi_{u_{0}, 1}^{\left(s_{1} s_{2} s_{3}\right)}=v^{6} .
\end{aligned}
$$

$G$ of type $C_{3}, p \neq 2$. (The simple reflection corresponding to the long root is denoted by $s_{3}$ and $\left(s_{1} s_{3}\right)^{2}=1 ; u_{2}^{\prime \prime}$ denotes a unipotent element which is regular inside a Levi subgroup of type $C_{2} ; u_{2}^{\prime}$ denotes a unipotent element with $\operatorname{dim} \mathcal{B}_{u_{2}^{\prime \prime}}=2$ which is not conjugate to $u_{2}^{\prime \prime}$.)

$$
\begin{aligned}
& \mathbf{S}_{u_{9}}=(1), \mathbf{S}_{u_{6}}=\left(s_{3}\right), \mathbf{S}_{u_{4}}=\left\{\left(s_{1}\right),\left(s_{2} s_{3} s_{2} s_{3}\right)\right\}, \mathbf{S}_{u_{3}}=\left\{\left(s_{1} s_{3}\right),\left(w_{0}\right)\right\}, \\
& \mathbf{S}_{u_{2}^{\prime}}=\left(s_{1} s_{2}\right), \mathbf{S}_{u_{2}^{\prime \prime}}=\left(s_{2} s_{3}\right), \mathbf{S}_{u_{1}}=\left(s_{2} s_{3} s_{1} s_{2} s_{3}\right), \mathbf{S}_{u_{0}}=\left(s_{1} s_{2} s_{3}\right) .
\end{aligned}
$$




$$
\begin{aligned}
& \Xi_{u_{9}, 1}^{1}=\left(1+v^{2}\right)^{3}\left(1+v^{4}\right)\left(1+v^{4}+v^{8}\right), \Xi_{u_{6}, 1}^{\left(s_{3}\right)}=v^{6}\left(1+v^{2}\right)^{2}\left(1+v^{4}\right), \\
& \Xi_{u_{4}, 1}^{\left(s_{2} s_{3} s_{2} s_{3}\right)}=v^{10}\left(v^{4}-1\right), \Xi_{u_{4}, 1}^{\left(s_{1}\right)}=2 v^{8}\left(1+v^{2}\right), \\
& \Xi_{u_{3}, 1}^{\left(s_{1} s_{3}\right)}=v^{8}\left(1+v^{2}\right), \Xi_{u_{3}, 1}^{\left(w_{0}\right)}=v^{14}\left(v^{4}-1\right), \Xi_{u_{2}^{\prime}, 1}^{\left(w_{1} s_{2}\right)}=v^{6}\left(1+v^{2}\right), \\
& \Xi_{u_{2}^{\prime \prime}, 1}^{\left(s_{2} s_{3}\right)}=v^{6}\left(1+v^{2}\right), \Xi_{u_{1}, 1}^{\left(s_{2} s_{3} s_{1} s_{2} s_{3}\right)}=v^{10}, \Xi_{u_{0}, 1}^{\left(s_{1} s_{2} s_{3}\right)}=v^{6} .
\end{aligned}
$$

1.2. We expect that the following property of $G$ holds:

(a)

$$
\underline{\mathbf{W}}=\sqcup_{u} \mathbf{S}_{u}
$$

( $u$ runs over a set of representatives for the unipotent classes in $G$ ).

The equality $\mathbf{W}=\cup_{u} \mathbf{S}_{u}$ is clear since for a regular unipotent $u$ and any $w$ we have $\Xi_{u, 1}^{w}=v^{2 l(w)}$. Note that (a) holds for $G$ of rank $\leq 3$ if $p$ is not a bad prime for $G$ (see 1.1). We will show elsewhere that (a) holds for $G$ of type $A_{n}$ (any $p$ ) and of type $B_{n}, C_{n}, D_{n}(p \neq 2)$. When $G$ is simple of exceptional type, (a) should follow by computing the product of some known (large) matrices using 1.5(a).

Assuming that (a) holds we define a surjective map from $\underline{\mathbf{W}}$ to the set of unipotent classes in $G$ by

$$
C \mapsto \mathcal{C}
$$

where $C \in \underline{\mathbf{W}}$ and $\mathcal{C}$ is the unique unipotent class in $G$ such that $C \in \mathbf{S}_{u}$ for $u \in \mathcal{C}$.

We expect that when $p=0$ we have

$$
c_{u} \in \mathbf{S}_{u}
$$

where for any unipotent element $u \in G, c_{u}$ denotes the conjugacy class in $\mathbf{W}$ associated to $u$ in [KL, 9.1]. Note that (c) holds for $G$ of rank $\leq 3$ (see 1.1). (We have used the computations of the map in [KL, 9.1] given in [KL, §9], [S1], [S2].)

Note added on October 21, 2010. After this paper was submitted, property (a) was established (in good characteristic) in the author's paper "From conjugacy classes in the Weyl group to unipotent classes", to appear in Representation Theory (electronic).

1.3. Assume that $G=S p_{2 n}(\mathbf{k})$ and $p \neq 2$. The Weyl group $\mathbf{W}$ can be identified in the standard way with the subgroup of the symmetric group $S_{2 n}$ consisting of all permutations of $[1,2 n]$ which commute with the involution $i \mapsto 2 n+1-i$. We say that two elements of $\underline{\mathbf{W}}$ are equivalent if they are contained in the same conjugacy class of $S_{2 n}$. The set of equivalence classes in $\underline{\mathbf{W}}$ is in bijection with the set of partitions of $2 n$ in which every odd part appears an even number of times (to $C \in \underline{\mathbf{W}}$ we attach the partition which has a part $j$ for every $j$-cycle of an element of $C$ viewed as a permutation of $[1,2 n])$. The same set of partitions of $2 n$ indexes the set of unipotent classes of $G$. Thus we obtain a bijection between the set of equivalence classes in $\underline{\mathbf{W}}$ and the set of unipotent classes of $G$. In other words we obtain a surjective map $\phi$ from $\underline{\mathbf{W}}$ to the set of unipotent classes of $G$ whose fibres are the equivalence classes in $\underline{\mathbf{W}}$. We will show elsewhere that for any unipotent class $\mathcal{C}$ in $G$ we have $\phi^{-1}(\mathcal{C})=\mathbf{S}_{u}$ where $u \in \mathcal{C}$.

1.4. Recall that the set of unipotent elements in $G$ can be partitioned into "special pieces" (see [L5]) where each special piece is a union of unipotent classes exactly one of which is "special". Thus the special pieces can be indexed by the set of isomorphism classes of special representations of $\mathbf{W}$ which depends only on $\mathbf{W}$ as a Coxeter 
group (not on the underlying root system). For each special piece $\sigma$ of $G$ we consider the subset $\mathbf{S}_{\sigma}:=\sqcup_{\mathcal{C} \subset \sigma} \mathbf{S}_{\mathcal{C}}$ of $\underline{\mathbf{W}}$ (here $\mathcal{C}$ runs over the unipotent classes contained in $\sigma)$. We expect that each such subset $\mathbf{S}_{\sigma}$ depends only on the Coxeter group structure of $\mathbf{W}$ (not on the underlying root system). As evidence for this we note that the subsets $\mathbf{S}_{\sigma}$ for $G$ of type $B_{3}$ are the same as the subsets $\mathbf{S}_{\sigma}$ for $G$ of type $C_{3}$. These subsets are as follows:

$$
\begin{aligned}
& \{1\},\left\{\left(s_{1}\right),\left(s_{3}\right),\left(s_{2} s_{3} s_{2} s_{3}\right)\right\},\left\{\left(s_{1} s_{3}\right),\left(w_{0}\right)\right\},\left\{\left(s_{1} s_{2}\right)\right\} \\
& \left\{\left(s_{2} s_{3}\right),\left(s_{2} s_{3} s_{1} s_{2} s_{3}\right)\right\},\left\{\left(s_{1} s_{2} s_{3}\right)\right\} .
\end{aligned}
$$

1.5. Let $g \in G$ be a unipotent element and let $z \in \bar{Z}(g), w \in W$. We show how the polynomial $\Xi_{g, z}^{w}$ can be computed using information from representation theory. We may assume that $p>1$ and that $\mathbf{k}$ is the algebraic closure of the finite field $\mathbf{F}_{p}$. We choose an $\mathbf{F}_{p}$ split rational structure on $G$ with Frobenius map $F_{0}: G \rightarrow G$. We may assume that $g \in G^{F_{0}}$. Let $q=p^{m}$ where $m \geq 1$ is sufficiently divisible. In particular $F:=F_{0}^{m}$ acts trivially on $\bar{Z}(g)$ hence $\operatorname{cl}(g)^{F}$ is a union of $G^{F}$-conjugacy classes naturally indexed by the conjugacy classes in $\bar{Z}(g)$; in particular the $G^{F}$-conjugacy class of $g$ corresponds to $1 \in \bar{Z}(g)$. Let $g_{z}$ be an element of the $G^{F}$-conjugacy class in $c l(g)^{F}$ corresponding to the $\bar{Z}(g)$-conjugacy class of $z \in \bar{Z}(g)$. The set $\mathcal{B}_{g_{z}}^{w}$ is $F$-stable. We first compute the number of fixed points $\left|\left(\mathcal{B}_{g_{z}}^{w}\right)^{F}\right|$.

Let $\mathcal{H}_{q}=\overline{\mathbf{Q}}_{l} \otimes_{\mathbf{Z}\left[v, v^{-1}\right]} \mathcal{H}$ where $\overline{\mathbf{Q}}_{l}$ is regarded as a $\mathbf{Z}\left[v, v^{-1}\right]$-algebra with $v$ acting as multiplication by $\sqrt{q}$. We write $T_{w}$ instead of $1 \otimes T_{w}$. Let $\operatorname{Irr} \mathbf{W}$ be a set of representatives for the isomorphism classes of irreducible $\mathbf{W}$-modules over $\overline{\mathbf{Q}}_{l}$. For any $E \in \operatorname{Irr} \mathbf{W}$ let $E_{q}$ be the irreducible $\mathcal{H}_{q}$-module corresponding naturally to $E$. Let $\mathcal{F}$ be the vector space of functions $\mathcal{B}^{F} \rightarrow \overline{\mathbf{Q}}_{l}$. We regard $\mathcal{F}$ as a $G^{F}$-module by $\gamma: f \mapsto f^{\prime}, f^{\prime}(B)=f\left(\gamma^{-1} B \gamma\right)$ for all $B \in \mathcal{B}^{F}$. We identify $\mathcal{H}_{q}$ with the algebra of all endomorphisms of $\mathcal{F}$ which commute with the $G^{F}$-action, by identifying $T_{w}$ with the endomorphism $f \mapsto f^{\prime}$ where $f^{\prime}(B)=\sum_{B^{\prime} \in \mathcal{B}^{F} ;\left(B, B^{\prime}\right) \in \mathcal{O}_{w}} f(B)$ for all $B \in$ $\mathcal{B}^{F}$. As a module over $\overline{\mathbf{Q}}_{l}\left[G^{F}\right] \otimes \mathcal{H}_{q}$ we have canonically $\mathcal{F}=\oplus_{E \in \operatorname{Irr} \mathbf{W}} \rho_{E} \otimes E_{q}$ where $\rho_{E}$ is an irreducible $G^{F}$-module. Hence if $\gamma \in G^{F}$ and $w \in \mathbf{W}$ we have $\operatorname{tr}\left(\gamma T_{w}, \mathcal{F}\right)=\sum_{E \in \operatorname{Irr} \mathbf{W}} \operatorname{tr}\left(\gamma, \rho_{E}\right) \operatorname{tr}\left(T_{w}, E_{q}\right)$. From the definition we have $\operatorname{tr}\left(\gamma T_{w}, \mathcal{F}\right)=$ $\left|\left\{B \in \mathcal{B}^{F} ;\left(B, \gamma B \gamma^{-1}\right) \in \mathcal{O}_{w}\right\}\right|=\left|\left(\mathcal{B}_{\gamma}^{w}\right)^{F}\right|$. Taking $\gamma=g_{z}$ we obtain

$$
\left|\left(\mathcal{B}_{g_{z}}^{w}\right)^{F}\right|=\sum_{E \in \operatorname{Irr} \mathbf{W}} \operatorname{tr}\left(g_{z}, \rho_{E}\right) \operatorname{tr}\left(T_{w}, E_{q}\right)
$$

The quantity $\operatorname{tr}\left(g_{z}, \rho_{E}\right)$ can be computed explicitly, by the method of [L4], in terms of generalized Green functions and of the entries of the non-abelian Fourier transform matrices [L2]; in particular it is a polynomial with rational coefficients in $\sqrt{q}$. The quantity $\operatorname{tr}\left(T_{w}, E_{q}\right)$ can be also computed explicitly (see [GP], Ch.10,11); it is a polynomial with integer coefficients in $\sqrt{q}$. Thus $\left|\left(\mathcal{B}_{g_{z}}^{w}\right)^{F}\right|$ is an explicitly computable polynomial with rational coefficients in $\sqrt{q}$. Substituting here $\sqrt{q}$ by $v$ we obtain the polynomial $\Xi_{g, z}^{w}$. (Here we use the trace formula for Frobenius maps.) This argument shows also that $\Xi_{g, z}^{w}$ is independent of $p$ (note that the pairs $(g, z)$ up to conjugacy may be parametrized by a set independent of $p$ ).

This is how the various $\Xi_{g, z}^{w}$ in 1.1 were computed, except in type $A_{1}, A_{2}, B_{2}$ where they were computed directly from the definitions. (For type $B_{3}, C_{3}$ we have used the computation of Green functions in [Sh]; for type $G_{2}$ we have used directly 
[CR] for the character of $\rho_{E}$ at unipotent elements.)

1.6. In this section we assume that $G$ is simply connected. Let $\tilde{G}=G(\mathbf{k}((\epsilon)))$ where $\epsilon$ is an indeterminate. Let $\tilde{\mathcal{B}}$ be the set of Iwahori subgroups of $\tilde{G}$. Let $\tilde{\mathbf{W}}$ be the affine Weyl group attached to $\tilde{G}$. Note that $\tilde{\mathbf{W}}$ naturally indexes $\left(w \mapsto \mathcal{O}_{w}\right)$ the orbits of $\tilde{G}$ acting on $\tilde{\mathcal{B}} \times \tilde{\mathcal{B}}$ by simultaneous conjugation on the two factors. For $g \in \tilde{G}$ and $w \in \tilde{\mathbf{W}}$ we set

$$
\tilde{\mathcal{B}}_{g}^{w}=\left\{B \in \tilde{\mathcal{B}} ;\left(B, g B g^{-1}\right) \in \mathcal{O}_{w}\right\} .
$$

By analogy with $[\mathrm{KL}, \S 3]$ we expect that when $g$ is regular semisimple, $\tilde{\mathcal{B}}_{g}^{w}$ has a natural structure of a locally finite union of algebraic varieties over $\mathbf{k}$ of bounded dimension and that, moreover, if $g$ is also elliptic, then $\tilde{\mathcal{B}}_{g}^{w}$ has a natural structure of algebraic variety over $\mathbf{k}$. It would follow that for $g$ elliptic and $w \in \tilde{\mathbf{W}}$,

$$
\Xi_{g}^{w}=\sum_{i, j \in \mathbf{Z}}(-1)^{i} \operatorname{dim} H_{c}^{i}\left(\tilde{\mathcal{B}}_{g}^{w}, \overline{\mathbf{Q}}_{l}\right)_{j} v^{j} \in \mathbf{Z}[v]
$$

is well defined; one can then show that the formal sum $\sum_{w \in \tilde{\mathbf{W}}} \Xi_{g}^{w} \hat{T}_{w}$ is central in the completion of the affine Hecke algebra consisting of all formal sums $\sum_{w \in \tilde{\mathbf{W}}} a_{w} \hat{T}_{w}$ $\left(a_{w} \in \mathbf{Q}(v)\right)$ that is, it commutes with any $\hat{T}_{w}$. (Here $\hat{T}_{w}$ is defined as in 1.1 and the completion of the affine Hecke algebra is regarded as a bimodule over the actual affine Hecke algebra in the natural way.)

\section{The sets $\mathrm{s}_{g}$.}

2.1. In this section we assume that $G$ is adjoint and $p$ is not a bad prime for $G$. For $g \in G, z \in \bar{Z}(g), w \in \mathbf{W}$ we set

$$
\xi_{g, z}^{w}=\left.\Xi_{g, z}^{w}\right|_{v=1}=\sum_{i \in \mathbf{Z}}(-1)^{i} \operatorname{tr}\left(z, H_{c}^{i}\left(\mathcal{B}_{g}^{w}, \overline{\mathbf{Q}}_{l}\right)\right) \in \mathbf{Z} .
$$

This integer is independent of $l$. For any $g \in G, z \in \bar{Z}(g)$ we set

$$
\pi_{g, z}=\sum_{w \in \mathbf{W}} \xi_{g, z}^{w} w \in \mathbf{Z}[W] .
$$

This is the specialization of $\Pi_{g, z}$ for $v=1$. Hence from 1.1(a) we see that $\pi_{g, z}$ is in the centre of the ring $\mathbf{Z}[\mathbf{W}]$. Thus for any $C \in \underline{\mathbf{W}}$ we can set $\xi_{g, z}^{C}=\xi_{g, z}^{w}$ where $w$ is any element of $C$. For $g \in G$ let $\mathbf{s}_{g}$ be the set of all $C \in \underline{\mathbf{W}}$ such that $\xi_{g, z}^{C} \neq 0$ for some $z \in \bar{Z}(g)$ and $\xi_{g^{\prime}, z^{\prime}}^{C}=0$ for any $g^{\prime} \in \overline{c l(g)}-c l(g)$ and any $z^{\prime} \in \bar{Z}\left(g^{\prime}\right)$. We describe the set $\mathbf{s}_{g}$ and the values $\xi_{g, z}^{C}=0$ for $C \in \mathbf{s}_{g}, z \in \bar{Z}(g)$, for various $G$ of low rank and various unipotent elements $g$ in $G$. We use the notation in 1.1. Moreover in the case where $\bar{Z}(g) \neq\{1\}$ we denote by $z_{n}$ an element of order $n$ in $\bar{Z}(g)$.

$G$ of type $A_{1}$.

$$
\mathbf{s}_{u_{1}}=(1), \mathbf{s}_{u_{0}}=\left(s_{1}\right) ; \xi_{u_{1}, 1}^{1}=2, \xi_{u_{0}, 1}^{s_{1}}=1
$$

$G$ of type $A_{2}$.

$$
\begin{gathered}
\mathbf{s}_{u_{3}}=(1), \mathbf{s}_{u_{1}}=\left(s_{1}\right), \mathbf{s}_{u_{0}}=\left(s_{1} s_{2}\right) . \\
\xi_{u_{3}, 1}^{1}=6, \xi_{u_{1}, 1}^{\left(s_{1}\right)}=1, \xi_{u_{0}, 1}^{\left(s_{1} s_{2}\right)}=1 .
\end{gathered}
$$


$G$ of type $B_{2}$.

$$
\begin{gathered}
\mathbf{s}_{u_{4}}=(1), \mathbf{s}_{u_{2}}=\left(s_{1}\right), \mathbf{s}_{u_{1}}=\left\{\left(s_{2}\right),\left(s_{1} s_{2} s_{1} s_{2}\right)\right\}, \mathbf{s}_{u_{0}}=\left(s_{1} s_{2}\right) . \\
\xi_{u_{4}, 1}^{1}=8, \xi_{u_{2}, 1}^{\left(s_{1}\right)}=2, \xi_{u_{1}, 1}^{\left(s_{2}\right)}=2, \xi_{u_{1}, 1}^{\left(s_{1} s_{2} s_{1} s_{2}\right)}=0 \\
\xi_{u_{1}, z_{2}}^{\left(s_{2}\right)}=0, \xi_{u_{1}, z_{2}}^{\left(s_{1} s_{2} s_{1} s_{2}\right)}=2, \xi_{u_{0}, 1}^{\left(s_{1} s_{2}\right)}=1 .
\end{gathered}
$$

$G$ of type $G_{2}$.

$$
\begin{gathered}
\mathbf{s}_{u_{6}}=(1), \mathbf{s}_{u_{3}}=\left(s_{2}\right), \mathbf{s}_{u_{2}}=\left(s_{1}\right), \mathbf{s}_{u_{1}}=\left\{\left(s_{1} s_{2} s_{1} s_{2} s_{1} s_{2}\right),\left(s_{1} s_{2} s_{1} s_{2}\right)\right\}, \mathbf{s}_{u_{0}}=\left(s_{1} s_{2}\right) . \\
\xi_{u_{6}, 1}^{1}=12, \xi_{u_{3}, 1}^{\left(s_{2}\right)}=2, \xi_{u_{2}, 1}^{\left(s_{1}\right)}=2, \xi_{u_{1}, 1}^{\left(s_{1} s_{2} s_{1} s_{2} s_{1} s_{2}\right)}=-3, \xi_{u_{1}, z_{2}}^{\left(s_{1} s_{2} s_{1} s_{2} s_{1} s_{2}\right)}=3, \\
\xi_{u_{1}, z_{3}}^{\left(s_{1} s_{2} s_{1} s_{2} s_{1} s_{2}\right)}=0, \xi_{u_{1}, 1}^{\left(s_{1} s_{2} s_{1} s_{2}\right)}=2, \xi_{u_{1}, z_{2}}^{\left(s_{1} s_{2} s_{1} s_{2}\right)}=0, \xi_{u_{1}, z_{3}}^{\left(s_{1} s_{2} s_{1} s_{2}\right)}=2, \xi_{u_{0}, 1}^{\left(s_{1} s_{2}\right)}=1 .
\end{gathered}
$$

$G$ of type $B_{3}$.

$$
\begin{aligned}
& \mathbf{s}_{u_{9}}=(1), \mathbf{s}_{u_{5}}=\left(s_{1}\right), \mathbf{s}_{u_{4}}=\left\{\left(s_{3}\right),\left(s_{2} s_{3} s_{2} s_{3}\right)\right\}, \mathbf{s}_{u_{3}}=\left(s_{1} s_{3}\right), \\
& \mathbf{s}_{u_{2}}=\left\{\left(s_{1} s_{2}\right),\left(w_{0}\right)\right\}, \mathbf{s}_{u_{1}}=\left\{\left(s_{2} s_{3}\right),\left(s_{2} s_{3} s_{1} s_{2} s_{3}\right)\right\}, \mathbf{s}_{u_{0}}=\left(s_{1} s_{2} s_{3}\right) . \\
& \xi_{u_{9}, 1}^{1}=48, \xi_{u_{5}, 1}^{\left(s_{1}\right)}=4, \xi_{u_{4}, 1}^{\left(s_{2} s_{3} s_{2} s_{3}\right)}=0, \xi_{u_{4}, z_{2}}^{\left(s_{2} s_{3} s_{2} s_{3}\right)}=4, \xi_{u_{4}, 1}^{\left(s_{3}\right)}=8, \\
& \xi_{u_{4}, 1}^{\left(s_{3}\right)}=0, \xi_{u_{3}, 1}^{\left(s_{3} s_{3}\right)}=2, \xi_{u_{2}, 1}^{\left(w_{0}\right)}=0, \xi_{u_{2}, z_{2}}^{\left(w_{0}\right)}=6 \\
& \xi_{u_{2}, 1}^{\left(s_{1} s_{2}\right)}=2, \xi_{u_{2}, z_{2}}^{\left(s_{1} s_{2}\right)}=0, \xi_{u_{1}, 1}^{\left(s_{2} s_{3}\right)}=2, \xi_{u_{1}, z_{2}}^{\left(s_{2} s_{3}\right)}=0, \\
& \xi_{u_{1}, 1}^{\left(s_{2} s_{3} s_{1} s_{2} s_{3}\right)}=0, \xi_{u_{1}, z_{2}}^{\left(s_{2} s_{3} s_{1} s_{2} s_{3}\right)}=2, \xi_{u_{0}, 1}^{\left(s_{1} s_{2} s_{3}\right)}=1 .
\end{aligned}
$$

$G$ of type $C_{3}$.

$$
\begin{aligned}
& \mathbf{s}_{u_{9}}=(1), \mathbf{s}_{u_{6}}=\left(s_{3}\right), \mathbf{s}_{u_{4}}=\left\{\left(s_{1}\right),\left(s_{2} s_{3} s_{2} s_{3}\right)\right\}, \mathbf{s}_{u_{3}}=\left(s_{1} s_{3}\right), \\
& \left.\mathbf{s}_{u_{2}^{\prime}}=\left(s_{1} s_{2}\right), \mathbf{s}_{u_{2}^{\prime \prime}}=\left(s_{2} s_{3}\right), \mathbf{s}_{u_{1}}=\left\{\left(s_{2} s_{3} s_{1} s_{2} s_{3}\right), w_{0}\right\} \mathbf{s}_{u_{0}}\right)=\left(s_{1} s_{2} s_{3}\right) . \\
& \xi_{u_{9}, 1}^{1}=48, \xi_{u_{6}, 1}^{\left(s_{3}\right)}=8, \xi_{u_{4}, 1}^{\left(s_{2} s_{3} s_{2} s_{3}\right)}=0, \xi_{u_{4}, z_{2}}^{\left(s_{2} s_{3} s_{2} s_{3}\right)}=4, \\
& \xi_{u_{4}, 1}^{\left(s_{1}\right)}=4, \xi_{u_{4}, 1}^{\left(s_{1}\right)}=0, \xi_{u_{3}, 1}^{\left(s_{1} s_{3}\right)}=2, \xi_{u_{2}^{\prime}, 1}^{\left(s_{1} s_{2}\right)}=2, \xi_{u_{2}^{\prime \prime}, 1}^{\left(s_{2} s_{3}\right)}=2, \\
& \xi_{u_{1}, 1}^{\left(s_{2} s_{3} s_{1} s_{2} s_{3}\right)}=1, \xi_{u_{1}, z_{2}}^{\left(s_{2} s_{3} s_{1} s_{2} s_{3}\right)}=1, \xi_{u_{1}, 1}^{\left(w_{0}\right)}=-3, \xi_{u_{1}, z_{2}}^{\left(w_{0}\right)}=3, \xi_{u_{0}, 1}^{\left(s_{1} s_{2} s_{3}\right)}=1 .
\end{aligned}
$$

2.2. For any unipotent element $u \in G$ let $n_{u}$ be the number of isomorphism classes of irreducible representations of $\bar{Z}(u)$ which appear in the Springer correspondence for $G$. Consider the following properties of $G$ :

$$
\underline{\mathbf{W}}=\sqcup_{u} \mathbf{s}_{u}
$$

( $u$ runs over a set of representatives for the unipotent classes in $G$ ); for any unipotent element $u \in G$,

$$
\left|\mathbf{s}_{u}\right|=n_{u} .
$$

The equality $\mathbf{W}=\cup_{u} \mathbf{s}_{u}$ is clear since for a regular unipotent $u$ and any $w$ we have $\xi_{u, 1}^{w}=1$. Note that (a),(b) hold in the examples in 2.1. We will show elsewhere that (a),(b) hold if $G$ is of type $A$. We expect that (a),(b) hold in general. 
Consider also the following property of $G$ : for any $g \in G, w \in \mathbf{W}$, $\xi_{g, 1}^{w}$ is equal to the trace of $w$ on the Springer representation

$$
\text { of } \mathbf{W} \text { on } \oplus_{i} H^{2 i}\left(\mathcal{B}_{g}, \overline{\mathbf{Q}}_{l}\right) \text {. }
$$

Again (c) holds if $G$ is of type $A$ and in the examples in 2.1; we expect that it holds in general. Note that in (c) one can ask whether for any $z, \xi_{g, z}^{w}$ is equal to the trace of $w z$ on the Springer representation of $\mathbf{W} \times \bar{Z}(g)$ on $\oplus_{i} H^{2 i}\left(\mathcal{B}_{g}, \overline{\mathbf{Q}}_{l}\right)$; but such an equality is not true in general for $z \neq 1$ (for example for $G$ of type $B_{2}$ ).

\section{REFERENCES}

[CR] B. Chang And R. Ree, The characters of $G_{2}(q)$, Istituto Naz. di Alta Mat. Symposia Math., XIII (1974), pp. 395-413.

[DL] P. Deligne and G. Lusztig, Representations of reductive groups over finite fields, Ann. Math., 103 (1976), pp. 103-161.

[GP] M. GeCk AND G. PfeIfFER, Characters of finite Coxeter groups and Iwahori-Hecke algebras, Clarendon Press Oxford, 2000.

[KL] D. Kazhdan and G. Lusztig, Fixed point varieties on affine flag manifolds, Isr. J. Math., 62 (1988), pp. 129-168.

[L1] G. Lusztig, On the reflection representation of a finite Chevalley group, in Representation theory of Lie groups, LMS Lect. Notes Ser. 34, Cambridge U. Press, 1979, pp. 325-337.

[L2] G. Lusztig, Unipotent representations of a finite Chevalley group of type $E_{8}$, Quart. J. Math., 30 (1979), pp. 315-338.

[L3] G. Lusztig, Character sheaves, I, Adv. in Math., 56 (1985), pp. 193-237.

[L4] G. Lusztig, On the character values of finite Chevalley groups at unipotent elements, J. Alg., 104 (1986), pp. 146-194.

[L5] G. Lusztig, Notes on unipotent classes, Asian J. Math., 1 (1997), pp. 194-207.

[Sh] T. ShoJI, On the Green polynomials of Chevalley groups of type $F_{4}$, Comm. in Alg., 10 (1982), pp. 505-543.

[S1] N. Spaltenstein, Polynomials over local fields, nilpotent orbits and conjugacy classes in Weyl groups, Astérisque, 168 (1988), pp. 191-217.

[S2] N. Spaltenstein, On the Kazhdan-Lusztig map for exceptional Lie algebras, Adv. Math, 83 (1990), pp. 48-74. 